\title{
Segundas líneas en cáncer de ovario ¿hay un estándar?
}

\author{
A. González Martín, M. Murcia, M. A. Vaz, E. López Miranda, M. López García
}

\section{Resumen}

La recaída del cáncer de ovario es habitualmente incurable y el tratamiento se basa en el empleo de quimioterapia paliativa. En pacientes con recaídas consideradas platinosensibles (más de 6 meses tras la última dosis de platino) existe suficiente evidencia científica para recomendar una combinación de quimioterapia basada en carboplatino, pues se ha demostrado un impacto en la supervivencia. Por el contrario, en las pacientes con progresión a platino (refractarias) o con recaídas antes de 6 meses (resistentes) el tratamiento recomendado es la monoterapia secuencial con los fármacos activos en segunda línea. La ausencia de tratamiento estándar en este contexto hace que la elección se deba basar en criterios de toxicidad, calidad de vida y preferencia de la paciente.

Palabras clave: Recaída de cáncer de ovario. Segunda línea de cáncer de ovario. Platino sensibilidad. Platino resistencia.

Oncología, 2007; 30 (1):3-11 


\section{Summary}

Recurrent ovarian carcinoma is usually incurable and palliative chemotherapy is the treatment of choice. In those patients considered as platinum-sensitive (with a platinum-free interval over 6 months) we have enough evidence to recommend carboplatin-based combination chemotherapy, due to the impact in survival observed in clinical trials comparing carboplatin monotherapy versus combination chemotherapy. On the other band, in those patient with progression to a platinum based chemotherapy (refractory) or with relapse in a period of less than 6 months (resistant), sequential monotherapy with active drugs in the second line is the recommended approach. The absence of a standard drug in this context makes that the choice should be based on toxicity criteria, quality of life and the preference of the patient.

Key words: Recurrent ovarian cancer. Second line. Platinum sensitive. Platinum resistant.

\section{Introducción}

El cáncer de ovario sólo representa el 3\% de las neoplasias diagnosticadas en la mujer, sin embargo es el responsable del $6 \%$ de las muertes por cáncer lo que le sitúa en la $4^{\text {a }}$ causa de muerte por cáncer en la mujer tras el cáncer de pulmón, mama y colorrectal $^{1}$. Varios factores influyen en la alta mortalidad de esta neoplasia, pero el más determinante es que la mayoría de pacientes (70-80\%) se diagnostican en estadios avanzados de la enfermedad.

El tratamiento estándar del cáncer de ovario avanzado consiste en la realización de una cirugía con el máximo esfuerzo citorreductor seguida de un tratamiento de quimioterapia intravenosa con carboplatino y un taxano, habitualmente paclitaxel ${ }^{2}$. Con este abordaje se consigue una remisión completa clínica en la mayoría de pacientes, pues se trata de una enfermedad quimiosensible. Sin embargo, la mayoría de las pacientes recaen y fallecen por la enfermedad. Incluso en las pacientes de mejor pronóstico, aquellas con una excelente cirugía que obtiene una citorreducción óptima inicial y son tratadas posteriormente con quimioterapia intraperitoneal, se observa una tasa de recaída del $50 \%$ a los 2 años y del $70 \%$ a los 4 años ${ }^{3}$.

La recaída del cáncer de ovario es habitualmente incurable, y el tratamiento tiene una intención esen-
TABLA I

\section{Objetivos del tratamiento de segunda línea del cáncer} de ovario

- Mejorar los síntomas relacionados con la enfermedad

- Alcanzar una respuesta objetiva del tumor

- Optimizar la calidad de vida global de las pacientes

- Retrasar el tiempo hasta la progresión de los síntomas

- Prolongar la supervivencia global

cialmente paliativa cuyos objetivos son controlar los síntomas relacionados con la enfermedad, mantener o mejorar la calidad de vida, aumentar el tiempo hasta la progresión y prolongar la supervivencia (Tabla I). Salvo algunos casos seleccionados que pueden ser subsidiarios de una cirugía de rescate ${ }^{4}$, el tratamiento de la recaída para la mayoría de pacientes consiste en quimioterapia. La gran heterogeneidad en el comportamiento clínico de las recaídas y la variedad de tratamientos de segunda línea disponibles, hacen que no se pueda estandarizar el tratamiento de la recaída ${ }^{2}$. Por ello para la elección del tratamiento hay que tener en cuenta un conjunto de factores dependientes de la enfermedad y de la paciente (Tabla II).

En ocasiones el único dato de recaída consiste en el ascenso del marcador tumoral CA-125 mientras 


\section{TABLA II}

Factores a considerar en la elección de segunda línea del cáncer de ovario

Dependientes del tratamiento:

- Respuesta a último tratamiento y tiempo transcurrido.

- Actividad y toxicidad de los tratamientos de segunda línea disponibles.

- Comodidad de administración y coste.

Dependientes de la paciente:

- Toxicidad experimentada con los tratamientos previos.

- Situación clínica de la paciente y enfermedades asociadas.

- Preferencia de la paciente.

que la paciente está asintomática sin evidencia de enfermedad clínica ni en las exploraciones radiológicas convencionales. Aunque nuevas tecnologías como el PET- TAC pueden cambiar esta situación ${ }^{5}$, el ascenso del CA-125 puede preceder la recaída clínicamente detectable una media de 2 a 6 meses, y hoy por hoy no existe evidencia de que iniciar el tratamiento antes de que se detecte clínicamente la enfermedad suponga un beneficio en la supervivencia para las pacientes.

\section{Factores pronóstico y predictores de respuesta}

La probabilidad de respuesta a una segunda línea en la recaída depende fundamentalmente de la respuesta previamente obtenida al tratamiento con platino y del tiempo transcurrido hasta la recaída, concepto denominado "intervalo libre de platino" (ILP). En un clásico estudio retrospectivo de Markman se observó que cuanto mayor era el intervalo libre de platino mayor era la probabilidad de respuesta a un nuevo esquema con platino ${ }^{6}$. Desde esta observación se han propuesto varias clasificaciones en función de la respuesta a la primera línea y del intervalo libre de platino (Tabla III $)^{7-10}$. La más usada por su sencillez y aplicabilidad clínica es la propuesta por Thigpen que sólo establece 2 grupos de pacientes: platino-sensibles, aquellas con respuesta inicial a un esquema de platino y recaída por encima de los 6 meses, y platino-resistentes, las que no tienen respuesta o recaen antes de 6 meses tras finalizar un tratamiento de primera línea con platino ${ }^{8}$. Sin embargo, otras clasificaciones como la propuesta en la guía NICE (Nacional Institute lor Health and Clinical Exce-

TABLA III

Clasificación de la recaída

\begin{tabular}{|llll|}
\hline \multirow{2}{*}{ Autor } & Mejor Respuesta a Platino & ILP & Taxonomía Platino \\
\hline Markman $^{7}$ & Progresión & - & Resistencia primaria \\
& No respuesta & cualquiera & Resistencia primaria \\
& Respuesta & $<6$ meses & Potencialmente sensible \\
& Respuesta & $>6$ meses & Potencialmente sensible \\
\hline Thigpen $^{8}$ & Progresión & - & Resistente \\
& No respuesta & cualquiera & Resistente \\
& Respuesta & $<6$ meses & Resistente \\
& Respuesta & $>6$ meses & Sensible \\
\hline \multirow{2}{*}{ Consenso $1998^{9}$} & Progresión & - & Refractaria \\
& No respuesta & Refractaria \\
& Respuesta & $<4$ meses & Refractario \\
& Respuesta & $>4-12$ meses & Intermedio \\
& Respuesta & $>12$ meses & Sensible \\
\hline NICE $2005^{10}$ & Progresión & - & Refractaria \\
& No respuesta & cualquiera & Refractaria \\
& Respuesta & $<6$ meses & Resistente \\
& Respuesta & $>6-12$ meses & Parcialmente sensible \\
& Respuesta & $>12$ meses & Sensible \\
\hline
\end{tabular}


llence) refleja mejor la heterogeneidad clínica de las pacientes en la recaída ${ }^{10}$.

Además del intervalo libre de platino, un estudio multivariante sobre 704 pacientes previamente tratadas con platino que recibieron posteriormente en segunda o tercera línea paclitaxel, docetaxel o epirubicina demostró que otros factores predictores de respuesta eran: un tamaño tumoral máximo $<5$ centímetros, no presentar más de 2 localizaciones tumorales y el tipo de histología serosa ${ }^{11}$.

Más recientemente se ha demostrado que la recaída con un patrón clínico predominante de carcinomatosis peritoneal es un factor pronóstico adverso (independiente del intervalo libre de platino) frente a un patrón de recaída en forma de lesiones concretas $^{12}$.

\section{Tratamiento de las pacientes con recaída platino sensible}

Desde los trabajos previamente citados de Markman, era un hecho conocido que las pacientes con recaídas de cáncer de ovario y un intervalo libre de platino de al menos 6 meses tras la primera línea podían volver a responder a tratamiento con platino. Siendo la probabilidad de respuesta tanto mayor cuanto mayor fuera este intervalo. En este contexto, la monoterapia con carboplatino ha sido para la mayoría el tratamiento de referencia para estas pacientes dada su facilidad de administración (una infusión de 30 minutos) junto con su aceptable perfil de toxicidad (ausencia de alopecia, náuseas y vómitos moderados, mielotoxicidad moderada y escasa neurotoxicidad).

Actualmente, disponemos de evidencia procedente de 2 estudios fase III que demuestran que la poliquimioterapia con una combinación de platino bien con paclitaxel o bien con gemcitabina es superior a la monoterapia con platino.

\section{Paclitaxel-carboplatino vs carboplatino}

El estudio ICON4, es el resultado del análisis combinado de 3 estudios paralelos desarrollados por el Medical Research Council (MRC) en el Reino Unido, el Grupo AGO en Alemania y el Instituto Mario Negri en Italia ${ }^{13}$. Aunque existieron algunos matices diferentes en los criterios de inclusión de los 3 estudios, se reclutaron 802 pacientes con recaída de cáncer de ovario que fueron randomizadas a una combinación de paclitaxel y platino (mayoritariamente con carboplatino) $(n=410)$ o un régimen de platino sin paclitaxel (mayoritariamente carboplatino) $(n=392)$. Todas las pacientes habían recibido previamente al menos una primera línea de platino y tenían un intervalo de recaída superior a 6 meses. De hecho, el 92\% sólo habían recibido una línea de tratamiento previa y el $75 \%$ tenía un intervalo libre de platino superior a 12 meses. Hay que destacar que sólo el $40 \%$ habían recibido un taxano en la primera línea.

Con una mediana de seguimiento de 42 meses se observó un beneficio en supervivencia libre de enfermedad (mediana de 12 meses vs 9 meses; HR: $0.77 ; 95 \%$ CI: $0.66-0.89 ; \mathrm{p}=0.0004)$ y en supervivencia global (mediana de 29 meses vs 24 meses; HR: 0.82; 95\%CI: 0.69-0.97; $\mathrm{p}=0.023$ ) a favor de la rama de paclitaxel-platino. En un análisis de subgrupos realizado a posteriori, se observó que el beneficio en supervivencia era más evidente en el grupo de pacientes con recaídas por encima de 12 meses y en las que no habían sido expuestas previal mente a taxanos. En cuanto a la tolerancia, aunque el esquema de combinación se asoció con mayor incidencia de toxicidad en forma de alopecia (86\%), y neuropatía periférica moderada-severa (20\%), no se observaron diferencias en el análisis de calidad de vida de las pacientes.

Un estudio fase II randomizado realizado por el Grupo GEICO (Grupo Español de Investigación en Cáncer de Ovario), arrojó unas conclusiones similares ${ }^{14}$. Se aleatorizaron 81 pacientes con recaída de cáncer de ovario platino sensible a paclitaxel 175 $\mathrm{mg} / \mathrm{m}^{2}$ más carboplatino AUC 5 frente a carboplatino AUC 5. Se observó un incremento en la tasa de respuesta (75\% vs 50\%; p 0.003), tiempo a progresión (12,2 meses versus 8,4 meses; log rank $\mathrm{p}=0.021)$ y supervivencia global a favor del esquema de combinación. A pesar de las limitaciones por el tamaño muestral, los resultados de este estudio se sumaron a la evidencia procedente del ensayo ICON4 de la superioridad de la combinación de paclitaxel-carboplatino sobre carboplatino.

Algunos autores han sugerido que un tratamiento secuencial con paclitaxel y carboplatino obtendría los mismos resultados que la combinación pero con menor toxicidad, hipótesis cuya confirmación requeriría el desarrollo de un ensayo clínico bien diseñado. 
TABLA IV

Estudios randomizados con monoterapia en cáncer de ovario recurrente

\begin{tabular}{|c|c|c|c|c|c|c|}
\hline Autor & $\begin{array}{c}\text { Med ILT } \\
\text { (meses) }\end{array}$ & Fármaco & $\begin{array}{c}N \\
(P R+P S)\end{array}$ & $\begin{array}{l}T R(\%) \\
(P R / P S)\end{array}$ & $\begin{array}{c}M T P(\text { semanas }) \\
(P R / P S)\end{array}$ & $\begin{array}{c}M S G \text { (semanas) } \\
(P R / P S)\end{array}$ \\
\hline Ten Bokkel Huinink ${ }^{16}$ & ¿? & $\begin{array}{l}\text { Topotecan } \\
\text { Paclitaxel }\end{array}$ & $\begin{array}{l}112(60+52) \\
114(59+55)\end{array}$ & $\begin{array}{c}20,5(13,3 / 28,8) \\
13,2(6,7 / 20)\end{array}$ & $\begin{array}{c}\mathbf{2 3} \\
14 \\
\mathrm{p}= \\
0.002\end{array}$ & $\begin{array}{l}61 \\
43\end{array}$ \\
\hline Gordon $^{17}$ & $\begin{array}{c}6,7 \\
7\end{array}$ & $\begin{array}{l}\text { Topotecan } \\
\text { DLP }\end{array}$ & $\begin{array}{l}235(124+111) \\
239(130+109)\end{array}$ & $\begin{array}{c}17(8 / 32) \\
19,7(16 / 31)\end{array}$ & $\begin{array}{c}17(13,6 / 23,3) \\
16,1(9,1 / 28,9) \\
\mathrm{p}=0,037 \text { para PS }\end{array}$ & $\begin{array}{c}56,7(41 / 71) \\
60(35 / \mathbf{1 0 8} \\
\mathrm{p}=0.008 \text { para PS }\end{array}$ \\
\hline $\mathrm{O}^{\prime} \mathrm{Byrne}^{18}$ & $\begin{array}{l}6,6 \\
6,7\end{array}$ & $\begin{array}{c}\text { DLP } \\
\text { Paclitaxel }\end{array}$ & $\begin{array}{l}106 \text { (40\% PS) } \\
107 \text { (37\% PS) }\end{array}$ & $\begin{array}{l}19 \\
24\end{array}$ & $\begin{array}{l}22 \\
22\end{array}$ & $\begin{array}{c}46,7 \\
56\end{array}$ \\
\hline Vermorken ${ }^{19}$ & $<12$ & $\begin{array}{l}\text { Oxaliplatin } \\
\text { Topotecan }\end{array}$ & $\begin{array}{l}79(51+28) \\
79(53+26)\end{array}$ & $\begin{array}{l}11,4(3,9 / 25) \\
8,9(5,7 / 15)\end{array}$ & $\begin{array}{l}- \\
-\end{array}$ & - \\
\hline Rosenberg $^{20}$ & ¿? & $\begin{array}{c}\text { Paclitaxel/sem } \\
\text { Paclitaxel/3 sem }\end{array}$ & $\begin{array}{l}104 \\
104\end{array}$ & $\begin{array}{l}31 \\
32\end{array}$ & $\begin{array}{l}5,5 \text { meses } \\
8,3 \text { meses }\end{array}$ & $\begin{array}{l}14,6 \text { meses } \\
19,2 \text { meses }\end{array}$ \\
\hline
\end{tabular}

Med ILT: mediana de intervalo libre de tratamiento; PR: platino-resistente; PS: platino-sensible; TR: tasa de respuesta; MTP: mediana de tiempo a progresión; MSG: mediana de supervivencia global; DLP: Doxorubicina Liposomal Pegilada.

\section{Gemcitabina-carboplatino vs carboplatino}

El estudio alemán AGO OVAR 2.5 coordinado por el grupo AGO con la participación de la EORTC-GCG (European Organization for Research and Treatment of Cancer Gynaecological Cancer Group) y el grupo canadiense del NCIC (Nacional Cancer Institute of Canada Clinical Trials Group) demostró que la combinación de carboplatino y gemcitabina era superior a carboplatino en la recaída platino-sensible, confirmando la superioridad de la poliquimioterapia con platino en este contexto ${ }^{15}$.

En este estudio se reclutaron 383 pacientes con recaída platino sensible que fueron aleatorizadas a la combinación de carboplatino AUC 4 día $1^{\circ}$ y gemcitabina $1000 \mathrm{mg} / \mathrm{m}^{2}$ días $1^{\circ}$ y $8^{\circ}$ frente a carboplatino AUC 5 en monoterapia. La combinación de carboplatino y gemcitabina fue superior en tasa de respuesta (47\% vs 31\%; $\mathrm{p}=0.0016)$ y supervivencia libre de progresión (HR 0.72; 95\% CI: 0.58-0.90) que era el objetivo principal del estudio. Un análisis de subgrupos mostró que la combinación beneficiaba tanto al grupo de pacientes con recaídas de menos de 12 meses como a las que habían recibido previamente paclitaxel en primera línea.
La toxicidad principal de este esquema fue la hematológica grado 3-4 consistente en: neutropenia (70\% de pacientes), anemia grado 3-4 (27\% de pacientes) y trombopenia grado 3-4 (35\% de pacientes). Aunque no resultó clínicamente relevante, dado que no se observaron diferencias en la incidencia de fiebre neutropénica o en el empleo de antibióticos, sí motivó el aumento en el uso de G-CSF y el requerimiento de transfusiones de hematíes. Por otra parte, la incidencia de neuropatía y alopecia fue muy baja.

Los resultados de este estudio han motivado la aprobación de gemcitabina en la recaída del cáncer de ovario platino sensible en asociación a carboplatino.

\section{Monoterapia no platino}

La década de los 90 asistió al desarrollo de nuevos fármacos con actividad en segunda línea de cáncer de ovario tras recaída a un tratamiento basado en platino. Entre estos fármacos hay que destacar por su actividad a: paclitaxel, topotecan, doxorrubicina lipososomal pegilada, oxaliplatino, docetaxel y gemcitabina.

Varios estudios fase III desarrollados en dicha década compararon estos fármacos en monoterapia entre sí en la segunda línea de tratamiento (Tabla IV), 
y sus resultados motivaron la aprobación de paclitaxel, topotecan y la doxorrubicina liposomal pegilada para el tratamiento de la segunda línea de cáncer de ovario ${ }^{16-18}$. De hecho son los tratamientos de uso habitual en pacientes con recaída platino-resistente como veremos más adelante.

Sin embargo, su aplicación en monoterapia en pacientes platino sensibles no puede ser recomendada hoy día a la luz de los conocimientos actuales. Hay que tener en cuenta que la interpretación y aplicabilidad de los estudios de registro se hace compleja en este momento debido fundamentalmente a dos motivos: 1) no todas las pacientes habían recibido el estándar actual de primera línea con paclitaxel-carboplatino, y 2) se incluyeron pacientes platino-resistentes y platino-sensibles.

Es cierto que el análisis de pacientes platino-sensibles muestra tasas de respuesta en torno al $30 \%$ con topotecan o doxorrubicina liposomal pegilada, y que esta última alcanza una mayor supervivencia que topotecan en este grupo de pacientes. Por este motivo se ha considerado una opción de tratamiento en pacientes con sensibilidad parcial a platino (aquellas con recaídas de 6-12 meses) ${ }^{21}$. Sin embargo, esta consideración tiene un sesgo importante pues nunca se han comparado estos fármacos con carboplatino que era el estándar en este contexto, y con el que se consiguen tasas de respuesta del 30$50 \%$. Además, es un hecho hoy reconocido que la poliquimioterapia con carboplatino es aún superior al carboplatino en monoterapia.

\section{Conclusiones}

Como conclusión de los estudios expuestos, podemos afirmar que la poliquimioterapia con platino debe ser el tratamiento de elección para las pacientes con recaída de cáncer de ovario platino sensible. El empleo de monoterapia no platino no está comparada con esquemas de poliquimioterapia por lo que no puede ser recomendada como tratamiento de elección.

La elección de una u otra combinación debe realizarse más teniendo en cuenta aspectos de toxicidad y preferencias de la paciente que a un particular análisis no planeado de subgrupos dentro de los estudios expuestos. La principal limitación de la combinación de paclitaxel-carboplatino es la neurotoxicidad y la alopecia. Dado que hoy día casi todas las pacientes reciben en primera línea dicha combinación, no es infrecuente que presenten cierto grado de neuropatía residual en la recaída que podría agravarse con el retratamiento con taxanos. La combinación de carboplatino y gemcitabina, con un diferente perfil de toxicidad principalmente hematológica, representa una alternativa en estas pacientes.

En búsqueda de una mejor tolerancia se han desarrollado otros dobletes de platino como la combinación de carboplatino y doxorrubicina liposomal pegilada $^{22}$. Los prometedores resultados con esta combinación han motivado la activación de un estudio fase III en el Gynecological Cancer InterGroup que compara paclitaxel $175 \mathrm{mg} / \mathrm{m}^{2}$ y carboplatino AUC 5 cada 3 semanas con carboplatino AUC 5 y doxorrubicina liposomal pegilada $30 \mathrm{mg} / \mathrm{m}^{2}$ cada 4 semanas (estudio CALYPSO).

\section{Tratamiento de las pacientes platino resistentes}

El grupo de pacientes platino resistentes se caracteriza por un pésimo pronóstico determinado por una limitada tasa de respuesta a la quimioterapia y una esperanza de vida que no supera los 10 meses. Lógicamente en este grupo de pacientes el objetivo fundamental del tratamiento es el control de los síntomas relacionados con la enfermedad, procurando no empeorar la calidad de vida de las pacientes por los efectos secundarios del tratamiento. Como se ha comentado previamente varios agentes desarrollados en los últimos 15 años han demostrado cierta actividad en pacientes resistentes con tasas de respuesta del 10 al 15\%. Entre los fármacos activos se encuentran paclitaxel, topotecan, doxorrubicina liposomal pegilada, docetaxel, gemcitabina, oxaliplatino, etopósido, ifosfamida, vinorelbina y hexametilmelamina. Dado que ningún fármaco se ha mostrado claramente superior en los ensayos fase III realizados (Tabla IV), la elección del tratamiento debe basarse en la comodidad de administración y la toxicidad esperada con el tratamiento, así como la preferencia de la paciente. Desde el punto de vista de coste-efectividad, la doxorrubicina liposomal pegilada resulta coste efectiva en este contexto comparada con topotecan y paclitaxe ${ }^{21}$.

En los últimos años se han publicado datos de estudios fase II con combinaciones de topotecan, pa- 
clitaxel, doxorrubicina liposomal pegilada y gemcitabina, que han mostrado tasas de respuesta algo superiores a las observadas en estudios fase III con monoterapia ${ }^{23-25}$. Sin embargo, ningún ensayo randomizado ha demostrado una superioridad de las combinaciones sobre la monoterapia ${ }^{26}$. El ejemplo más reciente ha sido un estudio presentado por el grupo alemán NOGGO en el congreso de ASCO de 2006, en el que se incluyeron 505 pacientes con recaída tras paclitaxel-platino que fueron randomizadas a topotecan monoterapia, topotecan-etopósido o topotecan-gemcitabina. No se observaron diferencias en los parámetros de supervivencia pero sí una mayor toxicidad con las combinaciones ${ }^{27}$.

Por todo lo anterior podemos concluir que, en ausencia de ensayos clínicos, el tratamiento de elección de las pacientes platino-resistentes continúa siendo el empleo secuencial de fármacos en monoterapia.

Mención aparte requiere el empleo de hormonoterapia en este grupo de pacientes. Varios ensayos clínicos han demostrado una tasa de respuesta en torno al $10 \%$ con Tamoxifeno en la recaída de cáncer de ovario. Esta respuesta es independiente de la expresión de receptores de estrógeno y progesterona. Dada la escasa toxicidad asociada a Tamoxifeno, este debe ser un fármaco a considerar en pacientes que no sean candidatas a quimioterapia o cuando se han agotado las distintas opciones terapéuticas. El interés por la hormonoterapia se ha renovado en el reciente congreso de ASCO 2006 por los resultados de dos estudios fase II con inhibidores de aromatasa. En un ensayo fase II con exemestano en una población de pacientes refractarias se observó una tasa de estabilizaciones duraderas (> 14 semanas) en el $36 \%$ de las pacientes ${ }^{28}$. En otro estudio se empleó letrozol en 46 pacientes que expresaban el receptor de estrógeno obteniendo una tasa de respuesta del $9 \%$ y un $42 \%$ de estabilizaciones en las 33 pacientes con enfermedad evaluable radiológicamente $^{29}$.

\section{Nuevos fármacos}

Al igual que en otras áreas de la oncología, en cáncer de ovario se está estudiando la eficacia de los nuevos agentes terapéuticos frente a las nuevas dianas biológicas, aunque hay que decir que el desa- rrollo de estos fármacos va por detrás del observado en otras patologías como el carcinoma de mama, colorrectal o de pulmón.

En estos momentos el fármaco cuyo desarrollo está más avanzado en cáncer de ovario es bevacizumab. En la edición de ASCO de 2005 se presentaron los datos de un estudio fase II del grupo GOG americano (Gynecologic Oncology Group) que demostró una tasa de respuesta del $17,7 \%$ en 62 pacientes con persistencia o recaída de cáncer de ovario y un máximo de 2 líneas de tratamiento previo ${ }^{30}$. Además, se observó una tasa de ausencia de progresión a 6 meses del $58 \%$, que es muy superior a la observada en otros estudios del GOG en este contexto. Estos prometedores resultados han motivado que se activen 2 ensayos fase III multicéntricos, uno americano (GOG 218) y otro dentro del grupo internacional GCIG (ICON7), en los que se explora el papel de bevacizumab en la primera línea. No obstante, el papel de bevacizumab en la segunda línea puede estar limitado por la alta tasa de perforaciones intestinales (hasta el 11,4\%). Dado que este evento se observa cuando se administra a pacientes con clínica o datos radiológicos de oclusión intestinal o engrosamiento de la pared intestinal, el empleo de bevacizumab en segundas o siguientes líneas requerirá una selección cuidadosa de las pacientes ${ }^{31}$.

A diferencia de otras patologías, curiosamente en cáncer de ovario existe aún interés en el desarrollo de nuevos agentes quimioterápicos para su empleo en segunda línea. Dos de los fármacos cuya actividad resulta más prometedora en la recaída son patupilona y trabectedina.

La patupilona es una epotilona natural cuyo mecanismo de acción citotóxico es la estabilización del microtúbulo, que ha demostrado actividad clínica en tumores sensibles y resistentes a taxanos. En un estudio fase I/II en 31 pacientes platino-resistentes se observó una tasa de respuesta bioquímica del $28 \%$, además se observaron 2 respuestas completas, 1 respuesta parcial y 7 estabilizaciones en las 19 pacientes con enfermedad evaluable radiológicamente. Los principales efectos adversos de esta epotilona que se administra mediante infusión intravenosa cada 3 semanas son diarrea, astenia y neuropatía periférica moderada ${ }^{32}$. Estos resultados han motivado la puesta en marcha de un estudio fase III en el que se compara la patupilona con la doxorrubicina liposomal pegilada en pacientes platino-resistentes. 
Trabectedina (Yondelis ${ }^{\mathrm{TM}}$ ) es un compuesto derivado marino cuyo mecanismo de acción es la unión al surco menor del ADN lo que distorsiona el ADN e induce una apoptosis independiente de p53. Trabectedina ha demostrado una tasa de respuestas próxima al $30 \%$ en pacientes con recidiva platino-sensible tanto en su administración en 3 horas, en infusión de 24 horas o en esquema semanal ${ }^{33-34}$. Actualmente un estudio fase III compara la combinación de trabectedina con doxorrubicina liposomal pegilada (DLP) frente a DLP en monoterapia en pacientes con recaídas antes de 12 meses.

\author{
Correspondencia: \\ Dr. A. González Martín \\ Servicio de Oncología Médica \\ Hospital Ramón y Cajal \\ Ctra. Colmenar Viejo, Km. 9,100 \\ E-28034 Madrid \\ agonzalezm@seom.org
}

9. Advanced epithelial ovarian cancer: 1998 consensus statements. Ann Oncol 1999; 10 (suppl 1): S87-S92.

10. National Institute for Clinical Excellence. Final Appraisal Determination. Ovarian cancer (advanced)-topotecan, pegylated liposomal doxorubicin hydrochloride and paclitaxel for second-line or subsequent treatment (review). www.nice.org.uk

11. Eisenhauer EA, Vermorken JB, van Glabbeke M. Predictors of response to subsequent chemotherapy in platinum pretreated ovarian cancer: a multivariate analysis of 704 patients. Ann Oncol 1997; 8: 963-8.

12. Ferrandina $G$ et al. Impact of pattern of recurrence on clinical outcome of ovarian cancer patients: Clinical considerations, European Journal of Cancer 2006, doi: 10.1016/j.ejca.2006.03.025.

13. The ICON and AGO Collaborators. Paclitaxel plus platinum-based chemotherapy versus conventional platinumbased chemotherapy in women with relapsed ovarian cancer: the ICON4/AGO-OVAR-2.2 trial. Lancet 2003; 361: 2099-106.

14. González-Martín A, Calvo E, Bover I, et al. Randomised phase II study of carboplatin versus paclitaxel-carboplatin in platinum-sensitive recurrent advanced ovarian cancer: a GEICO study. Ann Oncol 2005; 16:749-755.

15. Ptisterer J, Plante M, Vergote I, et al. Gemcitabine/carboplatin vs carboplatin in platinum sensitive recurrent ovarian cancer. Results of a Gynecologic Cancer Intergroup randomized phase III trial of the AGO OVAR, the NCIC CTG and the EORTC GCG. J Clin Oncol 2004; 22:5005 (suppl) (abst).

16. Ten Bokkel Huinink W, Gore M, Carmichael J, et al. Topotecan versus paclitaxel for the treatment of recurrent epithelial ovarian cancer. J Clin Oncol 1997; 15: 21832193.

17. Gordon AN, Tonda M, Sun S, et al. Long-term survival advantage for women treated with pegylated liposomal doxorubicin compared with topotecan in a phase 3 randomized study of recurrent and refractory ovarian cancer. Gynecol Oncol 2004; 95:1-8.

18. O'Byrne et al. A phase III study of Caelyx versus paclitaxel in platinum-treated taxane-naive relapsed ovarian cancer. Proc Am Soco Clin Oncol 2002; 21, 203a (abstract 808).

19. Vermorken J, Gore M, Perren T, et al. Multicenter randomised phase II study of oxaliplatin or topotecan in platinum-pretreated epithelial ovarian cancer patients. Proc Am Soco Clin Oncol 2001; 20, 212a (abstract 847).

20. Rosenberg P, Anderson H, Boman K, et al. A randomised multicenter study of single agent paclitaxel given weekly versus every three weeks to patients with ovarian cancer previously treated with platinum therapy. Proc Am Soco Clin Oncol 1999; 18, 368a (abstract 1420).

21. Main C, Bojke L, Griffin S, Norman G, Barbieri M, Mather L, et al. Topotecan, pegylated liposomal doxorubicin hydrochloride and paclitaxel for second-line or subsequent treatment of advanced ovarian cancer: a systematic review 
and economic evaluation. Health Technol Assess 2006; 10(9).

22. Ferrero J, Weber B, Lepille D, et al. Caelyx and carboplatin in patients with advanced ovarian cancer in late relapse (> 6 months): Late results a GINECO phase II trial. J Clin Oncol, 2004 ASCO Annual Meeting Proceedings (PostMeeting Edition). Vol 22, No 14S (July 15 Supplement): 5022.

23. García AA, O'Meara A, Bahador A, et al. Phase II study of gemcitabine and weekly paclitaxel in recurrent platinumresistant ovarian cancer. Gynecol Oncol 2004; 93(2):4938.

24. D’Agostino G, Ferrandina G, Ludovisi M, et al. Phase II study of liposomal doxorubicin and gemcitabine in the salvage treatment of ovarian cancer. Br J Cancer 2003; 89 (7):1180-1184.

25. Sehouli J, Stengel D, Oskay G, et al. Dose finding study for combination treatment with topotecan and gemcitabine of patients with recurrent ovarian cancer after failure of first-line chemotherapy with paclitaxel and platinum. Onkologie 2004; 27(1):58-64.

26. Bolis G, Parazzini F, Scarfone G, et al. Paclitaxel vs epidoxorubicin plus paclitaxel as second-line therapy for platinum-refractory and resistant ovarian cancer. Gynecol Oncol 1999; 72(1):60-4.

27. Sehouli J, Sommer H, Klare P, et al. Randomized multicenter phase III trial of topotecan monotherapy versus topotecan + etoposide versus topotecan + gemcitabine for second-line treatment of recurrent ovarian cancer. J Clin Oncol 2006 ASCO Annual Meeting Proceedings Part I. Vol 24, No. 18S (June 20 Supplement), 2006: 5030.

28. Yerma S, Alhayki M, Le T, et al. Phase II study of exemestane (E) in refractory ovarian cancer (ROE). J Clin On- col 2006. ASCO Annual Meeting Proceedings Part I. Vol 24, No. 18S (June 20 Supplement), 2006: 5026.

29. Gourley C, Smyth JF, Mackean M, et al. Phase II study of letrozole in estrogen receptor (ER) positive relapsed epithelial ovarian cancer. J Clin Oncol 2006 ASCO Annual Meeting Proceedings Part I. Vol 24, No. 18S (June 20 Supplement), 2006: 5025.

30. Burger RA, Sill M, Monk BJ, et al. Phase II trial of bevacizumab in persistent or recurrent epithelial ovarian cancer (EOC) or primary peritoneal cancer (PPC): a Gynecologic Oncology Group (GOG) study. J Clin Oncol 2005 ASCO Annual Meeting Proceedings. Vol 23, No. 16S, Part I of II (June 1 Supplement), 2005: 5009.

31. Cannistra S, Matulonis U, Penson R, et al. Bevacizumab in patients with advanced platinum-resistant ovarian cancer. J Clin Oncol 2006 ASCO Annual Meeting Proceedings Part I. Vol 24, No. 18S (June 20 Supplement), 2006: 5006.

32. Smit WM, Sufliarsky J, Spanik S, et al. Phase I/II dose-escalation trial of patupilone every 3 weeks in patients with relapsed/refractory ovarian cancer. J Clin Oncol 2005 ASCO Annual Meeting Proceedings. Vol 23, No. 16S, Part I of II (June 1 Supplement), 2005; 5056.

33. Del Campo J, Roszak A, Ciuleanu T, et al. Phase II open label randomized study of trabectedin $(\mathrm{T})$ given as two different dosing schedules in women with platinum-sensitive, recurrent ovarian carcinoma: Preliminary results. J Clin Oncol 2006. ASCO Annual Meeting Proceedings Part I. Vol 24, No. 18S (June 20 Supplement), 2006: 5031.

34. McMeekin DS, Krasner C, Chan S. Final results of a phase II study of weekly trabectedin in second/third line ovarian carcinoma. J Clin Oncol 2005 ASCO Annual Meeting Proceedings. Vol 23, No. 16S, Part I of II (June 1 Supplement), 2005: 5011. 